\title{
The Effect of Reverse Strain on Microstructure and Strengthening of Copper Fabricated by Severe Plastic Deformation of Torsion Process
}

\author{
Chengpeng WANG ${ }^{1,4}$, Fuguo LI ${ }^{2}$, Junkai FAN $^{3}$, Juncheng LIU ${ }^{1 *}$ \\ ${ }^{1}$ School of Materials Science and Engineering, Tianjin Polytechnic University, Tianjin 300387, PR China \\ ${ }^{2}$ School of Materials Science and Engineering, Northwestern Polytechnical University, Xi'an 710072, PR China \\ ${ }^{3}$ School of Mechanical and Power Engineering, Henan Polytechnic University, Jiaozuo 454000, PR China \\ ${ }^{4}$ School of Materials Science and Engineering, Taiyuan University of Technology, Taiyuan 030024, PR China
}

crossref http://dx.doi.org/10.5755/j01.ms.24.3.18414

Received 20 June 2017; accepted 16 November 2017

\begin{abstract}
The unidirectional single torsion process of commercially pure copper was followed by different number of reverse turns of torsion deformation. The effect of reverse strain on the material refinement and hardening was investigated. It is found that the grain refinement is significantly blocked in the reverse torsion strain in comparison with that only suffered in monotonic torsion strain. The strengthening slightly decreases with the torsional direction change. This phenomenon is interpreted in terms of the average dislocation density. A qualitative assumption is proposed to explain the retarded phenomenon of material refinement and hardening in the reverse torsion process. The reverse strain maybe improves the uniformity and stress-strain equilibrium of severe plastic deformation induced material.

Keywords: severe plastic deformation, copper, torsion, dislocation; hardness.
\end{abstract}

\section{INTRODUCTION}

Interest in the fabrication of ultrafine-grained (UFG) and nanomaterials through the severe plastic deformation (SPD) technique has attracted much attention recently [1]. SPD, as an extension of the traditional plastic deformation, is a very promising way of refining of material grain. However, the study on the subsequent stability and defects of UFG materials prepared by SPD is less, such as the residual stress, stabilization and homogeneity. Previous studies have found that different applied strain paths were essential to the grain refinement during the SPD processing [2]. Some earlier reports of loading paths of reverse highpressure torsion (HPT) [2-8] and equal channel angular pressing) (ECAP) [9-13] on the microstructure refinement and mechanical properties have pointed out the significant influence of deformation mode on SPD induced material. However, some revelations have not caught researchers' attention. The reverse strain could reduce the residual stress in the plastic deformed material. It is generally known that the residual stress reflects the distribution stress-strain in the interior of deforming material. This gives the authors enlightenments whether the change of deformation path can be used to improve the homogeneity and mechanical properties of the SPD induced material. Generally, torsion deformation has been recognized to be a kind of SPD method to obtain a higher plasticity strain than that in tension or compression [14]. As a representative example, HPT has an apparent and unique advantage in producing UFG materials with superior mechanical properties due to the employing a high compressive torsion deformation into the materials [14]. The torsion as a basic study is noticeable in the probe of

\footnotetext{
* Corresponding author. Tel.: +86-13682021846.

E-mail address: jchliu@tjpu.edu.cn (J. Liu)
}

SPD deformation behavior. So we adopted the reversal torsion for the investigation of reverse strain on the SPD induced material. Over the last century, the torsion studies were intense for the basic mechanics research [15-18]. But to date, very limited works referring to the torsion are available.

The present research was aimed to analyze the microstructural development and hardening during the reversal torsion, clarify the effect of reversal strain on the dislocation density, grain refinement of pure copper, and provide a mechanism explanation of the hardening and grain refinement in the reverse strain.

\section{EXPERIMENTAL PROCEDURES}

Prior to the reverse torsion, the commercial pure copper $(99.7 \%$ in wt.\%) had been preconditioned by annealing at $650{ }^{\circ} \mathrm{C}$ for $2 \mathrm{~h}$, and then by furnace cooling. Grain size and average Vickers microhardness was $100-200 \mu \mathrm{m}$ and $40 \pm 2 \mathrm{HV}$ for as-received samples. The torsion bars (see Fig. $1 \mathrm{a}$ and $\mathrm{b}$ ) with dimensions of $35 \mathrm{~mm}$ in gauge length and $5 \mathrm{~mm}$ in diameter were performed in wire torsion testing machine $\mathrm{XC}-10$ at a torsional rate of $0.25 \mathrm{turns} / \mathrm{min}$. As-receive samples were suffered in forward direction and subsequently in the reverse direction, and the torsion turn sum was up to 6 turns (see Fig. 1 a). In order to provide clear descriptions of the reverse torsion processing, we will designate the different combination as $\mathrm{x} 1 \mathrm{Fx} 2 \mathrm{R}$, where $\mathrm{x} 1$ and $\mathrm{x} 2$ were the turns of torsion, and the abbreviations of $\mathrm{F}$ and $\mathrm{R}$ represented the forward turn and reverse turn, respectively. For example, 1F5R denoted that the sample was strained to 1 turn of rotation in the forward direction and then suffered 5 turns of rotation in backward direction.

The equivalent strain $\varepsilon$ and shear strain $\gamma$ in torsion can be calculated as [14] 
$\gamma=2 \pi N r / l, \varepsilon=\gamma / \sqrt{3}$,

where $N$ is the number of rotations, $l$ and $r$ are the length and the distance from the center of samples, respectively.

According to the Eq. 1, the corresponding maximal shear strain and equivalent strain value for the 6 torsion turn at the surface of samples can be calculated as 2.69 and 1.55. Following the torsion process, selected copper samples were cut into segments and cold mounted in order to characterize the longitudinal and transverse sections (see Fig. 1 c). An optical microscope (OM) of Olympus PMG3 was used to observe the macrograph.

The microhardness was measured on a HXP-1000TM hardness testing machine with a load of $100 \mathrm{~g}$ and a loading time of $15 \mathrm{~s}$. Separated from each other by equal spaces of $\sim 0.25 \mathrm{~mm}$, the hardness indentation was impacted along the radial direction of the transverse section of samples, see Fig. 1 c. All the hardness values were averaged from at least 5 indentations for each measured point.

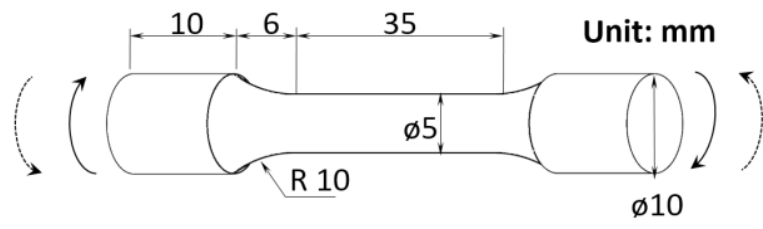

a

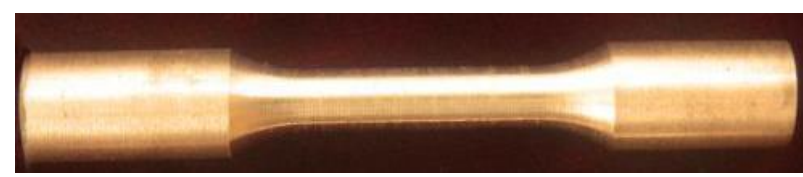

$\mathrm{b}$
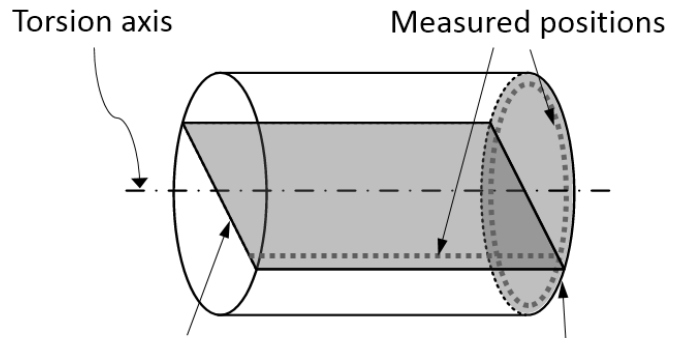

Longitudinal section

Transverse section

$\mathrm{c}$

Fig. 1. Schematic illustrations: a-different reversal torsion processes; $\mathrm{b}$ - pre-torsion samples; $\mathrm{c}$-measured positions

Microstructures of transmission electron microscopy (TEM) were characterized on a Tecnai G2 F30 operated at $300 \mathrm{KV}$. Cross-sectional thin foils for TEM observations were prepared by means of mechanical grinding and ion milling. The measurements of cell sizes were made directly from TEM observations and the reported values are averaged from a few hundred cells selected randomly. The cell size was measured along two axes of parallel and perpendicular. Approximately 100 cells were measured for each sample.

The evaluate dislocation density $\rho$ in the grains of the samples were observed by STEM with Ham's intersection method [3]. The first procedure for Ham's intersection method is a mesh drawn on a TEM image of a grain satisfying the diffraction condition, and then the number of intersections $m$ between the mesh and dislocations are counted. Eq. 2 is used to evaluate $\rho$ [4].

$\rho=2 m / L t$,

where $t$ is the thickness of the sample, $L$ is the total length of the measured mesh. To reduce the error of the invisible dislocations, TEM specimens were tilted $\left(0-2^{\circ}\right)$ in order to observe the highest number of dislocations within a grain.

\section{RESULTS AND DISCUSSION}

\subsection{Optical microstructure}

Optical microstructures of samples after torsion process are shown in Fig. 2. As shown in Fig. 2 a, an important feature of the inhomogeneous microstructure distribution is obvious in the central area (the left-hand side of the figure) and the edge region (the right-hand side of the figure). Grain refinement in the edge region is well due to the deformation of torsional simple shear. However, compared to the edge microstructure, the ones in the central region suffered less shear strain are relatively coarse. A similar phenomenon of inhomogeneous microstructure is also found in the microstructures of SPD induced materials processed by TE and HPT, etc. Due to the torsional deformation, a large number of elongated ultra-fine grains are parallel to the imposed shear direction (particularly in the microstructure of 1F5R and 5F1R samples). The banded textures as the typical shear textures are mainly caused by the increased strain sensitivity of slips. Despite a few of coarse residual annealing twins the grain refinement is obvious for the microstructure of $2 F 4 R$ and 4F2R samples. An intriguing phenomenon of vertically symmetrical metallographic distribution is seen in Fig. 2. Microstructure distribution of $2 F 4 R$ and 4F2R is symmetrical, and the microstructure distribution of $1 F 5 R$ and $5 \mathrm{~F} 1 \mathrm{R}$ is symmetrical. That is to say, the microstructural similarity is in the $2 F 4 R$ and $4 F 2 R$ samples. The 1F5R and 5F1R samples have also similar grain size and microstructure characteristic. The explanation is possibly attributed to the difference of the forward turns and reverse turns. For example, the difference of 2 turns is between in both $2 \mathrm{~F} 4 \mathrm{R}$ and $4 \mathrm{~F} 2 \mathrm{R}$ samples. According to the qualitative model of the average density of dislocations [2], the microstructure of 3F3R sample is abnormal course which may be caused by the dislocations annihilating. In addition, the refinement of 0F6R samples is more dominant than that of other torsion combinations. Similar results are in agreement with the results of previous HPT researches [2, 5-8]. To primarily demonstrate the refinement influence on the center region of microstructure, the symbolical small screenshots were captured from the metallographic figures of relatively coarse microstructure. The unique observation is shown in Fig. 2 b. According to the inhomogeneous microstructure distribution, the center region should be the area of symmetrical distribution for the circumferential area along the radial direction. The conspicuous microstructure observation of $3 \mathrm{~F} 3 \mathrm{R}$ sample is in agreement with the 
metallographic observation in Fig. 2 a. Both of the grain sizes are relatively consistent. This is determined by the inhomogeneous deformation behavior of torsion.

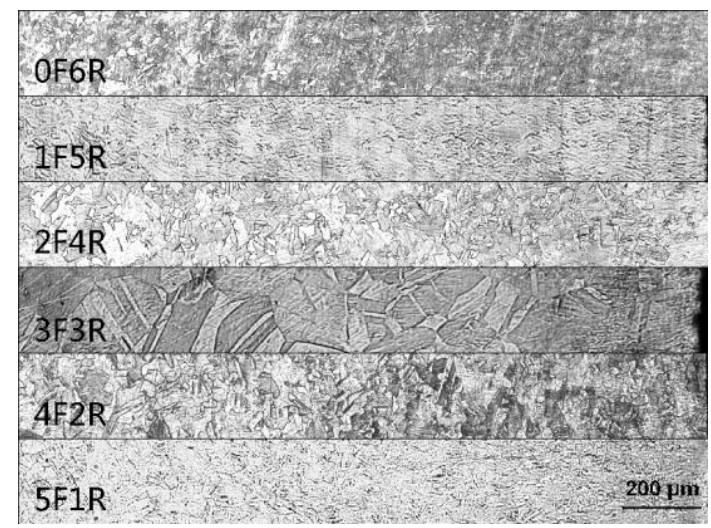

a

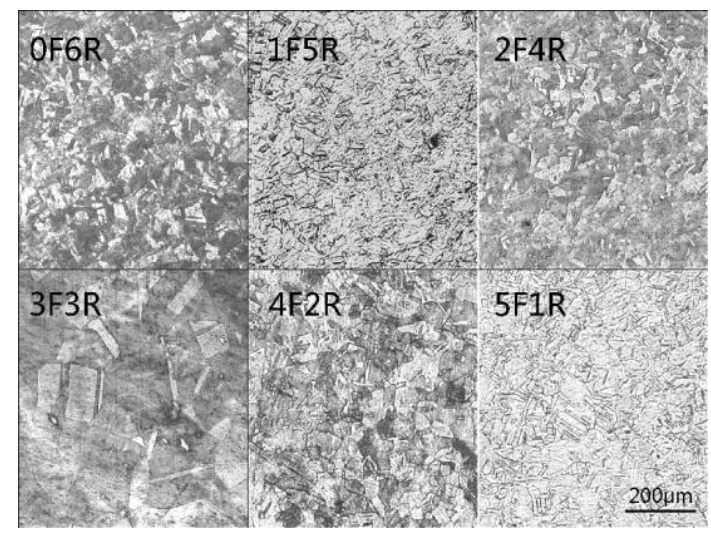

$\mathrm{b}$

Fig. 2. Optical microstructures of copper after torsion process: $\mathrm{a}$-longitudinal section; $\mathrm{b}$-center area of transverse section

In the torsion processing of cylindrical bar, if the angle of twist per unit length is sufficiently small, then the bar behaves linearly elastic. According to the theory of plasticity [19], if the angle of twist per unit length reaches a certain critical value, then some portion near the boundary of the bar becomes plastic. Studies indicated that the circular shaft was the strongest one under completely elastic or plastic torsion [19]. In general, the elastic-plastic torsion as a special case usually includes both the purely elastic and completely plastic torsion. Inverse torsion could release the elastic deformation energy of torsional material. The linear transmission of elastic and plastic deformation is reduced in the torsional deformation, which results in the difference decrease between the center area and edge area in the torsional deformed bar.

Moreover, residual stresses prevail throughout the inelastic (plastic) deformations, and the plastic torsion deformation is no exception. Mechanical processing method is a relief of residual stress in the metal plastic processing. An additional reversal deformation applied to the deformed products as the pressure secondary stress could reduce the residual stress value in the previous deformation. Reverse torsion would play such a role of reducing the extra inhomogeneous deformation in the internal material.

\subsection{TEM measurement}

Fig. 3 a shows the variation of dislocation density $\rho$ and cell sizes of the torsion processed samples. As can be seen from the figure, the symmetry is shown in the curves of dislocation density and cell sizes, and the symmetry axis lies in the position of 3R3F sample, which has the lowest vale of dislocation density and the highest value of cell sizes. The dislocation density $\rho$ decreases from $\sim 2.3 \times 10^{14} \mathrm{~m}^{-2}$ at $0 \mathrm{~F} 6 \mathrm{R} / 6 \mathrm{~F} 0 \mathrm{R}$ to the value of $\sim 1.2 \times 10^{14} \mathrm{~m}^{-2}$ at $3 \mathrm{~F} 3 \mathrm{R}$. Interestingly, the reversal torsion strain could reduce the saturation of $\rho$ in the torsion deformation. In figure, the cell size measured indicates a gradual decrease from $\sim 490 \mathrm{~nm}$ in $3 \mathrm{~F} 3 \mathrm{R}$ sample to $\sim 370 \mathrm{~nm}$ in $0 \mathrm{~F} 6 \mathrm{R} / 6 \mathrm{~F} 0 \mathrm{R}$ sample. Besides, cells become more equiaxed gradually with the decrease of difference value between the forward turn and reverse turn. This can be explained by the fact that the primary slip systems operating are parallel to the direction of the measurements taken parallel to the shear strain. The more slip systems operating with the compatible deformation in the microstructure evolution. Once the slip systems, perpendicular to the former slip systems, become activated, the length of these cells will be truncated. Dislocation cells have the certain extent reflection on the changes of slip system operating. Moreover, high-density dislocations based on different slip system movements, is impelled by the intense shear stress. When the dislocation density in the cell walls achieves a certain critical value, part of dislocations with different signs can annihilate at the cell boundaries. As a result, the thickness of dislocation wall becomes thin and the cell size decreases. As the dislocation cell size tends to a saturated value with continued plastic strain, a saturated grain size equivalent to the minimum cell size is expected with increasing strain. From the above, the reversal torsion strain could release part of the dislocation.

In order to explain the abnormal phenomenon of copper microstructure after the reverse torsion, the selective TEM obviation was analyzed in detail. Here, only the longitudinal TEM micrograph of sample $2 \mathrm{~F} 4 \mathrm{R}$ is shown in Fig. 3 b. For 2F4R, the original grains are clearly elongated to form shear bands (SBs) are present in Fig. 3 b. The SBs have a tendency to undergo a line up parallel to the shear direction during deformation. Accordingly, the formation of the shear bands originates from the deformation constraint imposed due to the torsion deformation pattern. This implies a large increase in the ability of dislocations to move over long distances. The increase in the ability of the dislocation motion is caused by the escape of dislocations from the tangles due to cross slip, which is responsible for plastic deformation at low strains. Moreover, the laminar shear structure (LSS) is shown inclined in the shear bands. The explanation should be due to the reverse torsion effect. The recovery occurs in the partial dislocations and reply slips. The fuzzy margin of LSS is caused by the unstable and inhomogeneous shear deformation. The microstructure diagram is shown in Fig. 3 c. Also, LSS in the SBs, a tendency to undergo an incline, follows the same relation as the recovery of shear strain in the forward turn. Based on the dislocation theory [14], the produced dislocations are hindered by the former 
generated ones with the strain increasing. So the dislocation motion is more and more difficult. Too many dislocations are formed as the entanglements, and cellular structures produce at a certain extent of strain. Dislocations densely tangle and mutually interfere in the cell wall area. The slip is difficult and makes the metal hardening. Thus, hardness increases with the increase of strain.

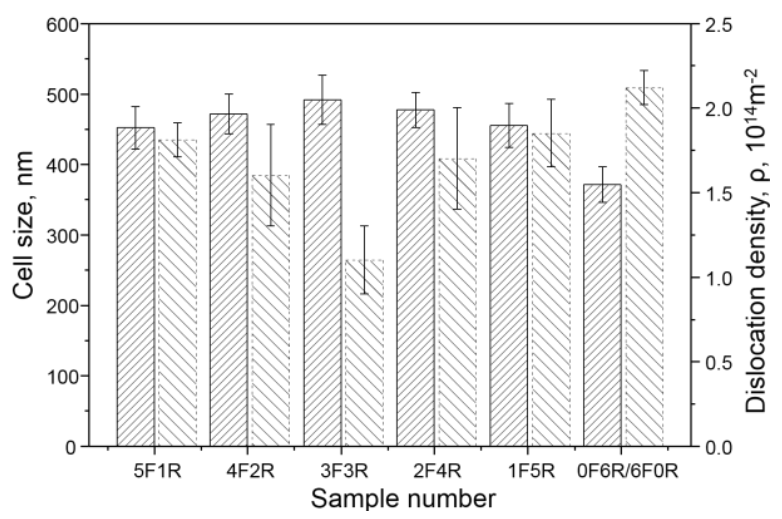

a

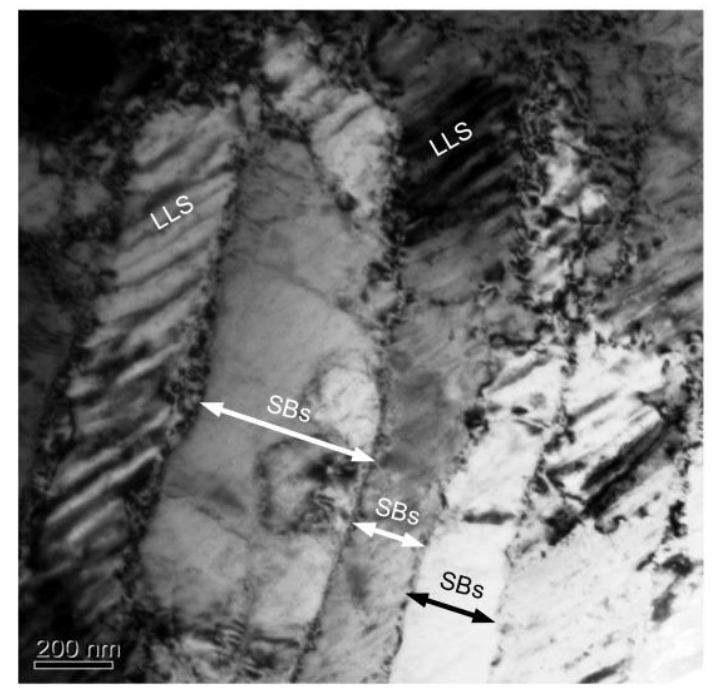

b

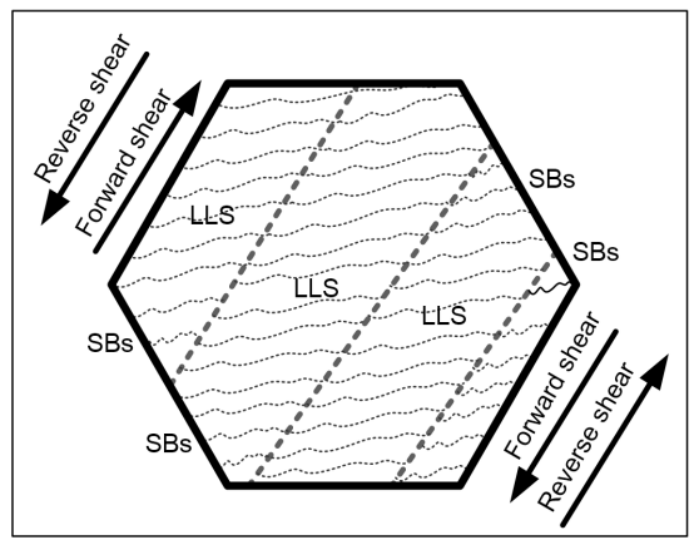

Fig. 3. a-variation of dislocation density and measured cell sizes for torsion processed pure copper; $b-$ TEM micrographs (bright field) of processed sample 2F4R; $\mathrm{c}$-schematic illustrations of microstructural evolution of $2 \mathrm{~F} 4 \mathrm{R}$ sample
The microstructures of longitudinal sections show elongated grains after the $2 \mathrm{~F} 4 \mathrm{R}$ torsion passes, but for the decreasing numbers of reverse passes, these elongated grains break down to relatively finer equiaxed grains.

\subsection{Microhardness examination}

Fig. 4 presents a comparison of the hardness variations of samples by different torsion procedures. In order to describe clearly, mathematical statistics and fitting were utilized to distinguish the different values. Added error bars using the user-specified $5 \%$ percentage, the values of measured points were fitted using the nonlinear curve fit of allometric1 model. With increasing the number of reversal turns, although all the curves show a similar trend, the hardness decreases with the increase of reversal torsion turns. There are some particulars in hardness distribution between monotonic and reversal deformation modes. Overall, the hardness value is arranged from large to small order as 0F6R, 1F5R and 5F1R, 2F4R, 4F2R, and 3F3R. Remarkably, either in the centre or in the periphery, the hardness of the samples processed by reversal torsion is even lower than by the monotonous torsion (sample 0F6R) process. These data show that reversal torsion is very inefficient in increasing hardness as compared to monotonous torsion process. Torsion like HPT produces substantial strain gradients resulting in both an inhomogeneous microstructure and the formation of dislocations [20, 21]. At the edge of the torsional sample, a high strain occurs. In this condition, the dislocations density is approximately proportional to the effective strain. The dislocations have symbolic dislocation characteristics of the local area, and thus the strain is significantly affected by the loading path. By that analogy, the monotonous torsion process improves the dislocations generation toward the center region of torsion sample. While the reverse turn dilutes this process due to the relieving of deformation energy and the next section goes into further detail. Comparison of data for the monotonous torsion processed samples show that the torsion turns in the opposite direction caused a drastic reduction in hardness over the entire sample, and the reduction of hardness is especially large in the lower hardness zone around the central region of the samples.

It should be noted that the hardness is plotted as a function of distance from the center $(r)$. The curves are roughly separated into two segments in accordance with the slope of the tangent line. The bifurcation point locates in the position of $r=1$. The first segment corresponds to the region of lower hardness near the center of the sample. Accordingly, the other presents a relatively high hardness. This could be due to the interaction of increasing dislocation density with the increasing radius, which becomes dominant after dislocation densities reach a certain critical value. After a total turn of one full revolution, the hardness appears to have reached a saturation level in the periphery of samples. As the amount of reverse turns increase, the increase of hardness gradually slows down considerably in processed samples. In the periphery of samples, the hardness is more homogeneous and shows a slight increase with increasing total turns up to the maximum of 6 turns. Also, this 
reduction/increment of hardness does not vary with the increasing radius except at and near the center.

The curve slopes of $0 \mathrm{~F} 6 \mathrm{R}, 1 \mathrm{~F} 5 \mathrm{R}$ and $5 \mathrm{~F} 1 \mathrm{R}$ samples have the same value at the center region of the traverse section. Three curves tend to overlap one another. The curve slope of 0F6R sample in the second segment is greater than the others. The curve slopes of $2 F 4 R, 4 F 2 R$, and 3F3R sample have the same value in the entire curve. That is why they are parallel to each other.

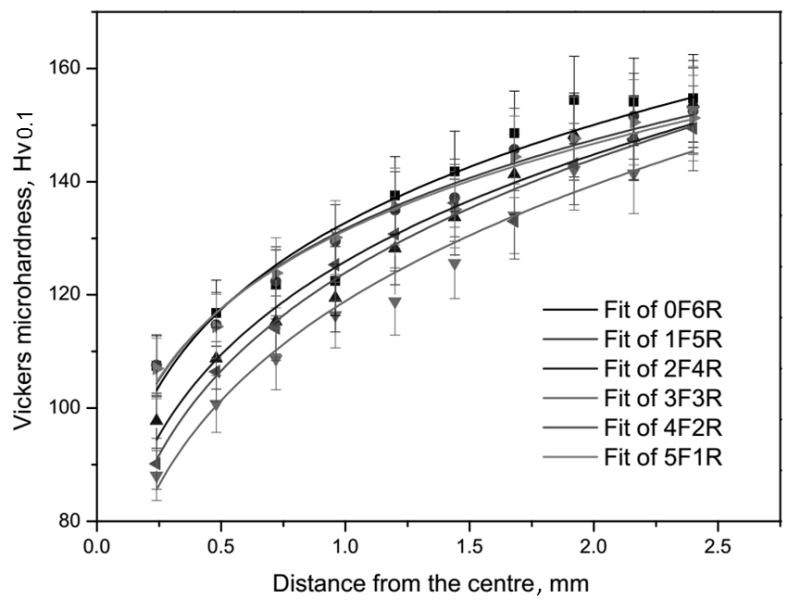

Fig. 4. Vickers microhardness vs. distance from the centre of samples

\subsection{A qualitative explication}

Fig. 5 shows the basic principles of the influence of dislocation creation on the reverse torsion processed material. Initially grain without dislocations is assumed a rectangle. First, take a look at the 0F6R or 6F0R samples (in Fig. 5 a), the lattice bends with the increasing torsion turns, and the density of dislocations in the initial grain also increases. When the dislocation reaches a critical value, these dislocations start to come together to form cell walls which evolve into subgrains/grain boundaries (GBs). For the monotonic torsion (without changing the direction of deformation) samples, the strengthening is mainly due to the high density of dislocations. For the 1F2R sample (Fig. 5 b), the configuration of the generated dislocations recovers due to the small torsional strain gradient (only one torsional turn) when the reverse torsion turns increases. However, the recovering configuration, different from the initial one, has a small number of dislocations. With the reverse torsion turn increasing, some cell walls evolve into subgrains/GBs, and some relatively few subgrains and dislocations are preserved comparing with the 0F6R or 6F0R samples (in Fig. 5 a). Similar case also exists in the 2F4R sample (in Fig. 5 c), whose microstructure is relatively coarse. The torsion in the opposite direction is applied to the grain, and this reversal strain gradient annihilates the dislocations initiating in the previous strain process, and as a result the refining effect weakens with a very low-density of dislocations. For the 3F3R sample (Fig. 5 d), refining and hardening are unsatisfactory due to the counteraction of forward turns and reverse turns. With the reverse turns increasing, the grain is subjected to a large strain gradient, and many dislocations generate and accumulate in the initial grain. Although the reverse strain gradient maybe destroys all the existing dislocations, the dislocations formed during the previous deformation should be relatively stable. During the small applied reverse strain, the dislocation density initially reduces to a very low level and subsequently increases again with the strain gradient further increasing. The recombined formations of dislocations, subgrains and GBs mainly depend on the annihilate magnitude of the reverse strain turns (Fig. 5 e and Fig. 5 f). The applied strain in torsion samples is closely related to the difference between the forward turns and reverse turns. Hence, similar macrostructure features and hardening are found in the foregoing analysis.
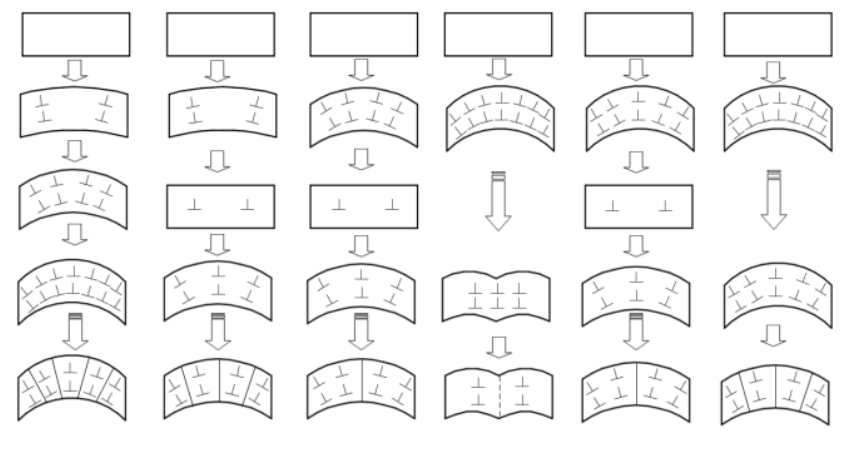

a

b

c

d

$\mathrm{e}$

$\mathrm{f}$

Fig. 5. Schematic illustration of the basic principles of the influence of dislocation creation on the microstructure of the reversal torsion processed samples: a $-0 \mathrm{~F} 6 \mathrm{R} / 6 \mathrm{~F} 0 \mathrm{R}$; b-1F5R; c-2F4R; d-3F3R; e-4F2R; f-5F1R

\section{CONCLUSIONS}

In the present study, the influence of reverse strain on hardening and refinement in the torsion process of commercially pure copper was investigated. The following main conclusions could be drawn:

1. The inhomogeneous microstructure exists in both unidirectional torsion and the reverse torsion. In addition, the reversal strain significantly affects the grain refinement in comparison with the monotonic straining.

2. The higher hardness is attained when the torsional straining is conducted by rotating the samples in the unidirectional direction. Less hardening is achieved if the torsional direction is reversed during the torsion processing.

3. The reverse strain could hinder the interior refinement and hardening of materials during the reverse strain in comparison with the monotonic techniques. However, the stress-strain in the material could be equilibrium under the action of reverse strain. The above phenomenon may be conducive to the performance improvement of SPD induced UFG material.

\section{Acknowledgements}

This work was partially supported by National Natural Science Foundation of China (No. 51275414, No. 51172161), School Youth Foundation (No. 120504020202), and the fund of the State Key Laboratory of Solidification Processing in NWPU (No. SKLSP201517). 


\section{REFERENCES}

1. Valiev, R.Z., $\quad$ Estrin, Y., $\quad$ Horita, Z., $\quad$ Langdon, T.G., Zehetbauer, M.J., Zhu, Y.T. Producing Bulk UltrafineGrained Materials by Severe Plastic Deformation JOM 58 (4) 2006: pp. $33-39$. https://doi.org/10.1007/s11837-006-0213-7

2. Zhang, J.W., Gao, N., Starink, M.J. Microstructure Development and Hardening During High Pressure Torsion of Commercially Pure Aluminium: Strain Reversal Experiments and a Dislocation Based Model Materials Science and Engineering: A 528 (6) 2011: pp. $2581-2591$. https://doi.org/10.1016/j.msea.2010.11.079

3. Miyajima, Y., Okubo, S., Abe, H., Okumura, H., Fujii, T., Onaka, S., Kato, M. Dislocation Density of Pure Copper Processed by Accumulative Roll Bonding and Equal-Channel Angular Pressing Mater Characterization 104 2015: pp. $101-106$. https://doi.org/10.1016/j.matchar.2015.04.009

4. Miyajima, Y., Mitsuhara, M., Hata, S., Nakashima, H., Tsuji, N. Quantification of Internal Dislocation Density Using Scanning Transmission Electron Microscopy in Ultrafine Grained Pure Aluminium Fabricated by Severe Plastic Deformation Materials Science and Engineering: A 528 (2) 2010: pp. 776-779. https://doi.org/10.1016/j.msea.2010.09.058

5. Horita, Z., Langdon, T.G. Microstructures and Microhardness of an Aluminum Alloy and Pure Copper After Processing by High-Pressure Torsion Materials Science and Engineering: A s 410-411(12) 2005: pp. $422-425$. https://doi.org/10.1016/j.msea.2005.08.133

6. Wetscher, F., Pippan, R. Cyclic High-pressure Torsion of Nickel and Armco Iron Philosophical Magazine 86 (36) 2006: pp. $5867-5883$. https://doi.org/10.1080/14786430600838288

7. Wetscher, F., Tian, B., Stock, R., Pippan, R. High Pressure Torsion of Rail Steels Mater Science Forum 503-504 2006: pp. 455-460.

https://doi.org/10.4028/www.scientific.net/MSF.503504.455

8. Todaka, Y., Umemoto, M., Yamazaki, A., Sasaki, J., Tsuchiya, K. Effect of Strain Path in High-Pressure Torsion Process on Hardening in Commercial Purity Titanium Materials Transactions 49 (1) 2008: pp. 47-53.

https://doi.org/10.2320/matertrans.ME200714

9. Li, S., Li, X., Yang, L. Role of Strain Path Change in Grain Refinement by Severe Plastic Deformation: A Case Study of Equal Channel Angular Extrusion Acta Materialia 61 (12) 2013: pp. 4398-4413. https://doi.org/10.1016/j.actamat.2013.04.010

10. Faria, C.G.D., $\quad$ Figueiredo, R.B., $\quad$ Aguilar, M.T.P., Cetlin, P.R. Strain Path Effects on the Development of Shear Bands During Shear Tests in Aluminum Alloy
Processed by ECAP Journal of Materials Research and Technology 4 (3) 2015: pp. 297-303.

https://doi.org/10.1016/j.jmrt.2015.01.009

11. Goto, M., Ando, Y., Han, S.Z., Kim, S.S., Kawagoishi, N., Euh, K. The Effect of Microstructural Inhomogeneity on the Growth Paths of Surface-Cracks in Copper Processed by Equal Channel Angular Pressing Engineering Fracture Mechanics 77 (11) 2010: pp. 1914-1925. https://doi.org/10.1016/j.engfracmech.2010.01.011

12. Huang, W.H., Yu, C.Y., Kao, P.W., Chang, C.P. The effect of Strain Path and Temperature on the Microstructure Developed in Copper Processed by ECAE Materials Science and Engineering: A 366 (2) 2004: pp. 221-228. https://doi.org/10.1016/j.msea.2003.08.033

13. Gholinia, A., Prangnell, P.B., Markushev, M.V. The Effect of Strain Path on the Development of Deformation Structures in Severely Deformed Aluminium Alloys Processed by ECAE Acta Materialia 48 (5) 2000: pp. $1115-1130$. https://doi.org/10.1016/S1359-6454(99)00388-2

14. Wang, C.P., Li, F.G., Wang, L., Yang, Y.J., Dong, J.Z. Experimental Microindentation of Pure Copper Subjected to Severe Plastic Deformation by Combined TensionTorsion Materials Science and Engineering: A 571 (4) 2013: pp. $95-102$. https://doi.org/10.1016/j.msea.2013.01.057

15. Nadai, A. Theory of Flow and Fracture of Solids. McGrawHill, New York, 1950: pp. 640-675.

16. Chakrabarty, J. Theory of Plasticity. ButterworthHeinemann, Oxford, 2006: pp. 84-86.

17. Estrin, Y., Tóth, L.S., Molinari, A., Bréchet, Y. A Dislocation-Based Model for All Hardening Stages in Large Strain Deformation Acta Materialia 46 (15) 1998: pp. 5509-5522. https://doi.org/10.1016/S1359-6454(98)00196-7

18. Fleck, N.A., Muller, H.M., Ashby, M.F., Hutchinson, J.W. Strain Gradient Plasticity: Theory and Experiment Acta Metallurgica Et Materialia 42 (2) 1994: pp. $475-487$. https://doi.org/10.1016/0956-7151(94)90502-9

19. Ting, T.W. Torsional Rigidities in the Elastic-Plastic Torsion of Simply Connected Cylindrical Bars Pacific Journal of Mathematics 46 (1) 1973: pp. 257-267. https://projecteuclid.org/euclid.pjm/1102946619

20. Aifantis, E.C. On the Microstructural Origin of Certain Inelastic Models Journal of Engineering Materials \& Technology 106 (4) 1984: pp. 326-330.

https://doi.org/doi:10.1115/1.3225725

21. Fleck, N.A., Hutchinson, J.W. A Phenomenological Theory for Strain Gradient Effects in Plasticity Journal of the Mechanics and Physics of Solids 41 (12) 1993: pp. $1825-1857$. https://doi.org/10.1016/0022-5096(93)90072-N 